\title{
Weights Guaranteeing Polefree Barycentric Rational Interpolation
}

Hamidreza Mofidi ${ }^{*}$ and Fazel Hadadifard ${ }^{2}$

'Department of Applied Mathematics, Tarbiat Modares University, Tehran, Iran; hamidreza.mofidi@gmail.com

${ }^{2}$ Department of Mathematics, University of Kansas, KS, US; f.hadadi@ku.edu

\begin{abstract}
The barycentric form of rational interpolants has some advantages among other representations [5]. Some authors have suggested many different kinds of weights ensuring that the rational interpolant written in barycentric form has no real poles. Here we give a necessary and sufficient condition for a rational interpolant written in barycentric form to have no poles when the nodes are located symmetrically relative to zero point. For some particular cases, some sets of suitable weights are also given.
\end{abstract}

Keywords: Barycentric Formula, Rational Interpolation, Interpolation.

Mathematics Subject Classification: 65D05; 65D15; 65D25.

\section{Introduction}

Let $f$ be a function defined on some closed interval $I$ of the real line, $x_{0}, x_{1}, \ldots, x_{n}$ be some $n+1$ distinct points in $I$, and $f_{j}=f\left(x_{j}\right), \quad j=0,1, \ldots, n$. Let $p_{n}(x)$ be the Lagrange representation of the unique polynomial of degree $n$, which interpolates $f$ on the points $x_{j}$ with $p_{n}\left(x_{j}\right)=f_{j}[16]$. Then

$$
p_{n}(x)=\sum_{j=0}^{n} l_{j}(x) f_{j},
$$

where,

$$
l_{j}(x)=\frac{\prod_{k=0, k \neq j}^{n}\left(x-x_{k}\right)}{\prod_{k=0, k \neq j}^{n}\left(x_{j}-x_{k}\right)}
$$

for $j=0,1, \ldots, n$ are the Lagrange polynomials. The barycentric formula for $p_{n}(x)$ has the following expression

$$
p_{n}(x)=\frac{\sum_{j=0}^{n} \frac{\omega_{j}}{x-x_{j}} f_{j}}{\sum_{j=0}^{n} \frac{\omega_{j}}{x-x_{j}}},
$$

where, $\omega_{j}$ is defined by [9]

$$
\omega_{j}=\frac{1}{\prod_{k=0, k \neq j}^{n}\left(x_{j}-x_{k}\right)}=\frac{1}{l^{\prime}\left(x_{j}\right)}, \quad j=0,1, \ldots, n
$$

and

$$
l(x)=\left(x-x_{0}\right)\left(x-x_{1}\right) \ldots\left(x-x_{n}\right) .
$$

As one should usually know, the polynomial interpolation suffers from two main drawbacks. The first one is that the polynomial interpolation may diverge for a particular set of nodes even for well-behaved functions [11]. The other problem is that even when we can choose nodes with a suitable distribution in the interval, the resulting polynomial interpolation may not be the expected answer or its convergence may happen to be too slowly for practical purposes [8]. Hence, as some common alternatives, one could use piecewise functions like splines [10] or rational interpolation, particularly with analytic functions $[1,22]$ Barycentric interpolation, first introduced in [21] through some simple changes in Lagrange representation of interpolation, has been gradually shown to be an appropriate representation of $p_{n}$. This representation

*Corresponding author:

Hamidreza Mofidi (hamidreza.mofidi@gmail.com) 
has been used by many authors like Henrici [16], Gautschi [14] and Werner [23] without mentioning the name explicitly. Among other related references, the paper by Berrut and Trefethen [9] discusses appropriately the barycentric representation of polynomial interpolation. The paper also gives a brief exposition of using the barycentric formulation for rational interpolation, trigonometric interpolation, spectral methods for differential equations, etc. We also refer the reader to see $[2,4,6,7$, $13,15,18]$ for further applications of the barycentric representation. Schneider and Werner were the first to notice the advantages of the barycentric representation in rational interpolation [20]. As briefly mentioned in section, the classical rational interpolation has some disadvantages, but they can often be resolved with the barycentric formula; the latter has several advantages investigated in papers such as [5] and [8] from which we repeat some parts.

The organization of paper is as follows. In section 2, a brief summary of classical rational interpolation and also some results concerning barycentric form of rational interpolation are given. Section 3, is devoted to our new idea and some theorems concerning barycentric weights. The new weights and some practical considerations are also discussed. In section 4 , some numerical experiments are considered.

\section{A Brief Summary of Barycentric Rational Interpolation}

The problem in classical rational interpolation is to find $r(x)=\frac{P(x)}{Q(x)}$, where $P \in P_{M}$ and $Q \in P_{N}$ such that

$$
r\left(x_{j}\right)=\frac{P\left(x_{j}\right)}{Q\left(x_{j}\right)}=f_{j}, \quad j=0,1, \ldots, n,
$$

where $P_{M}$ is the set of all polynomials with degree at most $M$. This problem has $M+N+2$ coefficients from $P$ and $Q$, one of which can be set to 1 appropriately. Therefore, one can consider $n=M+N$ in (5). This condition characterizes the classical rational interpolation problem [19]. The worst point is that there is no control over the occurrence of poles in the interval of interpolation [3]. In addition, the problem does not necessarily have a solution $[7,19]$, but it usually does, and in this case the solution is unique [19] and by Lemma 2.1, it can be written in barycentric form for some real values $u_{j}$ [5].
LeMmA 2.1: Let $\left\{\left(x_{j}, f_{j}\right)\right\}, j=0,1, \ldots, n$ be $(n+1)$ pairs of real numbers with $x_{j} \neq x_{k}, j \neq k$, and let $\left\{u_{j}\right\}$ be $n+1$ real numbers. Then

a) if $u_{k} \neq 0$, the rational function

$$
r(x)=\frac{\sum_{j=0}^{n} \frac{u_{j}}{x-x_{j}} f_{j}}{\sum_{j=0}^{n} \frac{u_{j}}{x-x_{j}}} \in \mathrm{R}_{n, n},
$$

interpolates $f_{k}$ at $x_{k}$.

b) conversely, every rational interpolant $r \in R_{n, n}$ of the given values $f_{j}$ may be written as in (6) for some $u_{j}^{\prime}$ s.

Proof: Notice that $R_{n, m}$ denotes the set of all rationals with numerator in $P_{n}$ and denominator in $P_{m}$. Considering $u_{j}=\omega_{j}$ in (6), rational interpolant $r(x)$ becomes the Lagrange interpolant $P_{n}$ in (2). And, one can generally see that $u_{j}=\omega_{j} Q\left(x_{j}\right)$, for rational function $\frac{P(x)}{Q(x)}$. We also have the following theorem on the occurrence of poles in the barycentric formula.

Theorem 2.1: Suppose the interpolation points have been ordered as $x_{0}<x_{1}<\ldots<x_{n}$ and the common factors in $r$ have been simplified to give the reduced function $\tilde{r}$ so that (6) corresponds to an interpolant with minimal denominator degree, and suppose $u_{j} \neq 0$ for all $j$. Then, if $\tilde{r}$ has no poles in $\left[x_{0}, x_{n}\right]$, then $\operatorname{sgn}\left(u_{j}\right)=-\operatorname{sgn}\left(u_{j+1}\right)$ for $j=0,1, \ldots, n-1$.

Proof: For the sake of simplicity, in the rest of this paper we assume that the function $r$ mentioned in (6) is actually the reduced function $\tilde{r}$ mentioned in the Theorem 2.1. It is also worth pointing out that this theorem provides a necessary condition for $r$ to have no poles. Berrut in [3] suggests and proves that with weights

$$
u_{j}=(-1)^{j}, \quad j=0,1, \ldots, n
$$

[6] is a rational function, with no pole in R. A generalization has been suggested by Floater and Hormann [12] They construct an interpolant by blending polynomials in the following way. For $d(0 \leq d \leq n)$ fixed, and for each $i=0,1, \ldots, n-d$, let $q_{\mathrm{i}}(x)$ denote the unique polynomial of degree at most $d$ that interpolates $f$ at the $d+1$ points $x_{i}, \ldots, x_{i+d^{*}}$. They consider

$$
r(x)=\frac{\sum_{j=0}^{n-d} \lambda_{i}(x) q_{i}(x)}{\sum_{j=0}^{n-d} \lambda_{i}(x)},
$$


where,

$$
\lambda_{i}(x)=\frac{(-1)^{i}}{\left(x-x_{i}\right) \ldots\left(x-x_{i+d}\right)} .
$$

This construction gives a family of rational interpolants, for $d=0,1, \ldots, n$ and it turns out that they have no real poles, and for $d \geq 1$ the interpolant has approximation order $O\left(h^{d+1}\right)$ as $h \rightarrow 0$, where $h=\max _{0 \leq i \leq n-1}\left(x_{i+1}-x_{i}\right)$ with $f(x) \in \mathrm{C}^{d+2}[a, b]$. The interpolant $r(x)$ in (7) is not appropriate for computation, but, according to Lemma 2.1, it can be expressed in barycentric form (6). The weights for the barycentric form $r(x)$ of (7) are given explicitly as

$$
u_{j}=\sum_{i \in J_{j}}(-1)^{i} \prod_{\substack{k=i \\ k \neq j}}^{i+d} \frac{1}{x_{j}-x_{k}}
$$

where,

$$
I=\{0,1, \ldots, n\}, \quad J_{j}=\{i \in I ; j-d \leq i \leq j\} .
$$

It is shown in [12] that $r(x)$ in (7) has no poles in $\mathrm{R}$, that the weights as defined by (8) alternate in sign.

\section{Some Theorems about Barycentric Weights and the New Weights}

It is observed from Theorem 2.1 that if for some $j, 0 \leq j \leq n-1, u_{j}$ and $u_{j+1}$ have the same sign, then $r(x)$ has at least one pole in the interval $\left(x_{j}, x_{j+1}\right)$. Therefore, if $r$ is reduced, alternating negative and positive signs of all $u_{j}^{\prime} s$ is a necessary condition for the denominator $r(x)$ to have no root. But this is not a sufficient condition to ensure that there will not be any poles for $r(x)$ [6]. However, in most related articles, after introducing some appropriate weights, they had to go into somehow complicated mathematics to prove that the denominator of $r(x)$, the corresponding barycentric interpolation (6) related to their introduced weights, has no root $[3,12]$. Like most articles in this field, the Chebyshev and Legendre or equally spaced nodes, we also suppose that the nodes belong to a symmetrical interval $[-a, a], a \in \mathrm{R}$,

$$
-a \leq x_{0}<\ldots<x_{n} \leq a,
$$

in a way that the nodes are located symmetrically relative to zero point, e.g. $x_{j}=-x_{n-j}$ for $j=0,1, \ldots, n$. We then rewrite the nodes as follows

$$
\left\{\begin{array}{ll}
x_{-\frac{N}{2}}, \ldots, x_{-1}, x_{0}=0, x_{1}, \ldots, x_{\frac{N}{2}} & N=n, n \text { even }, \\
x_{-\frac{N}{2}}, \ldots, x_{-1}, x_{1}, \ldots, x_{\frac{N}{2}}, & N=n+1, n \text { odd } .
\end{array}\right\}
$$

With this assumption, $x_{j}=-x_{n-j}, j=0, \ldots, \frac{N}{2}$, and $N$ is always even. If $n$ is even, $x_{0}=0$, otherwise the nodes will not contain $x_{0}$. According to the above assumptions and as multiplication of the weights by a nonzero real number does not change the interpolation formula in barycentric form, we then, for standardization purpose assume that

$$
\operatorname{sgn}\left(u_{j}\right)=(-1)^{j}, \quad j=0,1, \ldots, \frac{N}{2},
$$

and for $j=-1,-2, \ldots,-\frac{N}{2}$, we define

$$
u_{j}=\left\{\begin{array}{cc}
u_{-j}, & n \text { even } \\
-u_{-j}, & n \text { odd }
\end{array}\right.
$$

Obviously, (10) and (11) ensure that the considered weights $u_{j}^{\prime} s$ alternate in sign. Conditions, (9), (10) and (11) do not impose any limitation further than considered by other authors, except in a case when the $u_{i}$, which depends on $f$, are non-symmetric when $f$ itself is nonsymmetric. Hence, the following theorem which deals with a sufficient condition for weights is also applicable in their cases.

THEOREM 3.1: Let the nodes be as (9) and $u_{j}^{\prime}$ s satisfy (10) and (11). Then the denominator $r(x)$ of given by (6) and the polynomial $Z(x)$ given below are nonzero constant multiples of each other,

$$
Z(x)=\left\{\begin{array}{ll}
\frac{1}{2} u_{0} S_{0}(x)+\sum_{j=1}^{\frac{N}{2}} u_{j} S_{j}(x), N=n, \quad n \text { even, } \\
\sum_{j=1}^{\frac{N}{2}} u_{j} \bar{S}_{j}(x), & N=n+1, n \text { odd. }
\end{array}\right\}
$$

where,

$$
\begin{array}{ll}
S_{j}(x)=\prod_{k=0, k \neq j}^{\frac{N}{2}}\left(x^{2}-x_{k}^{2}\right), & j=0, \ldots, \frac{N}{2}, \\
\bar{S}_{j}(x)=\prod_{k=1, k \neq j}^{\frac{N}{2}}\left(x^{2}-x_{k}^{2}\right), & j=1, \ldots, \frac{N}{2} .
\end{array}
$$

Proof: We begin by rewriting $r(x)$ as follows

$$
r(x)=\frac{\sum_{j=-\frac{N}{2}}^{\frac{N}{2}}\left[\frac{u_{j}}{x-x_{j}} f_{j}\right]}{\sum_{j=-\frac{N}{2}}^{\frac{N}{2}}\left[\frac{u_{j}}{x-x_{j}}\right]}=\frac{\sum_{j=-\frac{N}{2}}^{\frac{N}{2}}\left[u_{j} f_{j} \prod_{k=-\frac{N}{2}, k \neq j}^{\frac{N}{2}}\left(x-x_{k}\right)\right]}{\sum_{j=-\frac{N}{2}}^{\frac{N}{2}}\left[u_{j} \prod_{k=-\frac{N}{2},}^{\frac{N}{2}}\left(x \neq j=x_{k}\right)\right]},
$$


where, for $n$ odd the zero index will not belong to indices of $\Pi$ and $\Sigma$ in (14). To continue the proof we now consider two cases. 1. $n$ even and $N=n$ : in this case for each $j=1,2, \ldots, \frac{N}{2}$ we
have,

$$
\begin{gathered}
u_{j} \prod_{k=-\frac{N}{2}, k \neq j}^{\frac{N}{2}}\left(x-x_{k}\right)+u_{-j} \prod_{k=-\frac{N}{2}, k \neq-j}^{\frac{N}{2}}\left(x-x_{k}\right) \\
=u_{j}\left(x+x_{j}\right) x \prod_{k=1, k \neq j}^{\frac{N}{2}}\left(x^{2}-x_{k}^{2}\right)+u_{j}\left(x-x_{j}\right) x \prod_{k=1, k \neq j}^{\frac{N}{2}}\left(x^{2}-x_{k}^{2}\right) \\
=2 u_{j} x^{2} \prod_{k=1, k \neq j}^{\frac{N}{2}}\left(x^{2}-x_{k}^{2}\right)=2 u_{j} \prod_{k=0, k \neq j}^{\frac{N}{2}}\left(x^{2}-x_{k}^{2}\right)=2 u_{j} S_{j}(x),
\end{gathered}
$$

and for $j=0$ we have,

$$
u_{0} \prod_{k=-\frac{N}{2}, k \neq 0}^{\frac{N}{2}}\left(x-x_{k}\right)=u_{0} \prod_{k=1}^{\frac{N}{2}}\left(x^{2}-x_{k}^{2}\right)=u_{0} S_{0}(x) .
$$

Replacing the above relations in the denominator of the rational function $r(x)$ yields

$$
\sum_{j=-\frac{N}{2}}^{\frac{N}{2}}\left(u_{j} \prod_{k=-\frac{N}{2}, k \neq j}^{\frac{N}{2}}\left(x-x_{k}\right)\right)=u_{0} S_{0}(x)+2 \sum_{j=1}^{\frac{N}{2}} u_{j} S_{j}(x)=2 Z(x),
$$

which confirms that in this case the denominator of $r(x)$ is a constant multiple of $Z(x)$.

2. $n$ odd and $N=n+1$ in this case for each $j=1,2, \ldots, \frac{N}{2}$ we have,

$$
\begin{aligned}
& u_{j} \prod_{k=-\frac{N}{2}, k \neq 0, j}^{\frac{N}{2}}\left(x-x_{k}\right)+u_{-j} \prod_{k=-\frac{N}{2}, k \neq 0,-j}^{\frac{N}{2}}\left(x-x_{k}\right) \\
= & u_{j} \quad\left(x+x_{j}\right) \prod_{k=1, k \neq j}^{\frac{N}{2}}\left(x^{2}-x_{k}^{2}\right)-u_{j}\left(x-x_{j}\right) \prod_{k=1, k \neq j}^{\frac{N}{2}}\left(x^{2}-x_{k}^{2}\right) \\
= & 2 x_{j} u_{j} \prod_{k=1, k \neq j}^{\frac{N}{2}}\left(x^{2}-x_{k}^{2}\right)=2 x_{j} u_{j} \bar{S}_{j}(x) .
\end{aligned}
$$

Therefore, after replacing the above relations into the denominator of $r(x)$ we again obtain

$$
\sum_{j=-\frac{N}{2}}^{\frac{N}{2}}\left(u_{j} \prod_{k=-\frac{N}{2}, k \neq j}^{\frac{N}{2}}\left(x-x_{k}\right)\right)=2 \sum_{j=1}^{\frac{N}{2}} x_{j} u_{j} \bar{S}_{j}(x)=2 Z(x) .
$$

Hence, the proof is complete.

Corollary 3.1: For the nodes as in (9) and $u j^{\prime} s$ satisfying (10) and (11), $r(x)$ given by (6) has no real poles if and only if the polynomial $Z(x)$ has no real roots. In addition, when $Z(x)$ has no real roots in the domain, its sign at each point in the domain will be $(-1)^{\frac{N}{2}}$.

Proof: According to Theorem 3.1, it is obvious that $r(x)$ has no real poles if and only if $Z(x)$ is nonzero everywhere. For the rest of proof we should prove that when $r(x)$ has no poles, the sign of $Z(x)$ at each point is $(-1)^{\frac{N}{2}}$. To do this, it suffices to determine its sign only at a point in the domain. For $\varepsilon>0$ consider the point $x_{i_{\varepsilon}}=x_{i}+\varepsilon$ in $\left(x_{i}, x_{i+1}\right)$. For each $k, k \neq i$

$$
S_{j}\left(x_{i_{\varepsilon}}\right)=\prod_{k=0, k \neq j}^{\frac{N}{2}}\left(x_{i_{\varepsilon}}^{2}-x_{k}^{2}\right)=\left(x_{i_{\varepsilon}}^{2}-x_{k}^{2}\right) \prod_{k=0, k \neq j, i}^{\frac{N}{2}}\left(x_{i_{\varepsilon}}^{2}-x_{k}^{2}\right) .
$$

From (15) it is observed that as $\varepsilon \rightarrow 0$, then $S_{j}\left(x_{i_{e}}\right) \rightarrow 0$, $j=0, \ldots, \frac{N}{2}, j \neq i$. Therefore, (12) confirms that when $\varepsilon$ is sufficiently close to zero, then for $n$ even, $Z\left(x_{i_{\varepsilon}}\right)$ and $u_{i} S_{i}\left(x_{i_{\varepsilon}}\right)$ have the same sign, and a similar situation also holds for $n$ odd and $\bar{S}_{j}(x)$. Obviously, for $n$ even and $N=n$, and $i=0,1, \ldots, \frac{N}{2}-1$, for $x \in\left(x_{i}, x_{i+1}\right)$, it can easily be seen

$$
\operatorname{sgn}\left(u_{j} S_{i}(x)\right)=(-1)^{i}(-1)^{\frac{N}{2}-i}=(-1)^{\frac{N}{2}},
$$

and for $n$ odd and $N=n+1$, and $i=0,1, \ldots, \frac{N}{2}-1$ for $x \in\left(x_{i}, x_{i+1}\right)$, we have

$$
\operatorname{sgn}\left(x_{i} u_{j} \bar{S}_{i}(x)\right)=(-1)^{i}(-1)^{\frac{N}{2}-i}=(-1)^{\frac{N}{2}} .
$$

Hence, from (16) and (17), the sign of $Z(x)$ at each point in the domain should be $(-1)^{\frac{N}{2}}$.

It should be noticed that by Theorem 3.1 and Corollary 3.1 the $u_{j}$ can be chosen in a way that the resulting barycentric rational function $r(x)$ has no real poles. What we do in Theorem 3.2 is a case in point. Before that, Lemma 3.1 provides us with what we need for proof of the Theorem.

LеммA 3.1: For the polynomials $S_{j}(x)$ defined by (13), $j=0,1, \ldots, \frac{N}{2}$, 
a) if $\gamma \in[0,2] \subseteq \mathrm{R}$, to be used as an exponent for $x$, then for $i=0,1, \ldots,\left(\frac{N}{2}-1\right)$ in intervals $\left(x_{i}, x_{i+1}\right)$ and $\left(x_{-(i+1)}, x_{-i}\right)$ we have

1. if $1 \leq j \leq i$ then

$$
\operatorname{sgn}\left(x_{j}^{\gamma} S_{j}(x)-x_{j-1}^{\gamma} S_{j-1}(x)\right)=(-1)^{\frac{N}{2}-i} .
$$

2. if $i+1 \leq j \leq \frac{N}{2}-1$ then

$$
\operatorname{sgn}\left(x_{j}^{\gamma} S_{j}(x)-x_{j+1}^{\gamma} S_{j+1}(x)\right)=(-1)^{\frac{N}{2}-i-1} .
$$

b) For each $j, j=0,1, \ldots, \frac{N}{2}-1$,

$$
x_{j}^{2} S_{j}(x)-x_{j+1}^{2} S_{j+1}(x)=x^{2}\left[S_{j}(x)-S_{j+1}(x)\right] .
$$

c) For each $j, j=0,1, \ldots, \frac{N}{2}$,

$$
\left.\left(1+x_{j}^{2}\right) S_{j}(x)-S_{0}(x)=(1+x)^{2}\right)\left[S_{j}(x)-S_{0}(x)\right]
$$

Proof: Our proof starts with this consideration that in intervals $\left(x_{i}, x_{i+1}\right)$ and $\left(x_{-(i+1)}, x_{-i}\right)$ we have

$$
\operatorname{sgn}\left(S_{j}(x)\right)= \begin{cases}(-1)^{\frac{N}{2}-i}, & 0 \leq j \leq i, \\ (-1)^{\frac{N}{2}-i-1}, & i+1 \leq j \leq \frac{N}{2} .\end{cases}
$$

a) For $1 \leq j \leq i$ we find,

$$
\operatorname{sgn}\left(\prod_{k=0, k \neq j, j-1}^{\frac{N}{2}}\left(x^{2}-x_{k}^{2}\right)=\operatorname{sgn}\left(S_{j}(x)\right)=\operatorname{sgn}\left(S_{j-1}(x)\right)=(-1)^{\frac{N}{2}-i}\right.
$$

and also

$$
\begin{aligned}
& \operatorname{sgn}\left(x_{j}^{\gamma} S_{j}(x)-x_{j-1}^{\gamma} S_{j-1}(x)\right)=\operatorname{sgn}\left(\prod_{k=0, k \neq j, j-1}^{\frac{N}{2}}\left(x^{2}-x_{k}^{2}\right)\right. \\
& \left.\left(x_{j}^{\gamma}\left(x^{2}-x_{j-1}^{2}\right)-x_{j-1}^{\gamma}\left(x^{2}-x_{j}^{2}\right)\right)\right) .
\end{aligned}
$$

For $i+1 \leq j \leq \frac{N}{2}-1$ we have,

$$
\operatorname{sgn}\left(\prod_{k=0, k \neq j, j+1}^{\frac{N}{2}}\left(x^{2}=x^{2}{ }_{k}\right)\right)=-\operatorname{sgn}\left(S_{j}(x)\right)=-\operatorname{sgn}\left(S_{j+1}(x)\right)=(-1)^{\frac{N}{2}-i} .
$$

To finish the proof of first part of case a), from (19) and (20), one needs to show that for each $j, 1 \leq j \leq i$, the term $x_{j}^{\gamma}\left(x^{2}-x_{j-1}^{2}\right)-x_{j-1}^{\gamma}\left(x^{2}-x_{j}^{2}\right)=x^{2}\left(x_{j}^{\gamma}-x_{j-1}^{\gamma}\right)+x_{j}^{\gamma} x_{j-1}^{\gamma}$ $\left(x_{j}^{2-\gamma}-x_{j-1}^{2-\gamma}\right)$ is always positive, which is true from our assumptions $0 \leq \gamma \leq 2$ and $0 \leq x_{j-1} \leq x_{j}$. Thus, the proof is completed. Similar argument can be followed for the proof of second part of $a$ ). We now turn to the proof of parts $b$ ) and $c$ ) of Lemma 3.1.

$$
\text { b) } \begin{aligned}
& x_{j}^{2} S_{j}(x)-x_{j+1}^{2} S_{j+1}(x)=\prod_{k=0, k \neq j, j+1}^{\frac{N}{2}}\left(x^{2}-x_{k}^{2}\right)\left[x_{j}^{2}\left(x^{2}-x_{j+1}^{2}\right)\right. \\
& \left.-x_{j+1}^{2}\left(x^{2}-x_{j}^{2}\right)\right] \\
= & \prod_{k=0, k \neq j, j+1}^{\frac{N}{2}}\left(x^{2}-x_{k}^{2}\right)\left[x^{2}\left[\left(x^{2}-x_{j+1}^{2}\right)-\left(x^{2}-x_{j}^{2}\right)\right]\right] \\
= & x^{2}\left(S_{j}(x)-S_{j+1}(x)\right) .
\end{aligned}
$$

c) $\left(1+x_{j}^{2}\right) S_{j}(x)-S_{0}(x)=\prod_{k=0, k \neq 0, j}^{\frac{N}{2}}\left(x^{2}-x_{k}^{2}\right)\left[\left(1+x_{j}^{2}\right) x^{2}\right.$

$$
\begin{aligned}
& \left.-\left(x^{2}-x_{j}^{2}\right)\right] \\
& =\prod_{k=0, k \neq 0, j}^{\frac{N}{2}}\left(x^{2}-x_{k}^{2}\right)\left[x_{j}^{2}\left(1+x^{2}\right)\right] \\
& =\left(1+x^{2}\right) \prod_{k=0, k \neq 0, j}^{\frac{N}{2}}\left(x^{2}-x_{k}^{2}\right)\left[x^{2}-\left(x^{2}-x_{j}^{2}\right)\right] \\
& =(1+x)^{2}\left[S_{j}(x)-S_{0}(x)\right] .
\end{aligned}
$$

TheOrem 3.2: Suppose that the nodes are given by (9) and $u j^{\prime}$ s satisfy (10) and (11) with absolute values

$$
\left|u_{j}\right|= \begin{cases}b_{0}+\sum_{l=1}^{m} b_{l} x_{j}^{\gamma_{l}}, & n \text { even, } \\ \sum_{l=1}^{\mathrm{m}} b_{l}^{\prime} x_{j}^{\gamma_{l}^{\prime}}, & n \text { odd },\end{cases}
$$

where $b_{l}$ and $b_{l}^{\prime}$ are nonnegative, $0 \leq \gamma_{l} \leq 2$ and $0 \leq \gamma_{l}^{\prime} \leq 1$ for each $l$. Moreover, suppose that $m \in N, b_{0}$ and $b_{l}^{\prime}$ are nonzero for at least one index $l$. Then with these weights, $r(x)$ has no real poles.

Proof: We prove for $n$ even and leave the proof for $n$ odd as it is similar. Suppose that $n$ is even, then by replacing $u j^{\prime}$ s in $Z(x)$, given by (12), after rearranging the terms of this summation in appropriate pairs like $(*)$ and $\left(*^{\prime}\right)$, we obtain

$$
\begin{aligned}
& Z(x)=\frac{1}{2} b_{0} S_{0}(x)+\sum_{k=1}^{\frac{N}{2}}(-1)^{k}\left(b_{0}+\sum_{(l=1)}^{m} b_{l} x_{k}^{\gamma_{l}}\right) S_{k}(x) \\
& =\left[\frac{1}{2} b_{0} S_{0}(x)-\cdots\right. \\
& +(-1)^{i-1}\left(\left(b_{0}+\sum_{l=1}^{m} b_{l} x_{i-1}^{\gamma_{l}}\right) S_{i-1}(x)\right)+(-1)^{i}\left(\left(b_{0}+\sum_{l=1}^{m} b_{l} x_{i}^{\gamma_{l}}\right) S_{i}(x)\right)
\end{aligned}
$$




$$
\begin{aligned}
& \frac{+(-1)^{i+1}\left(\left(b_{0}+\sum_{l=1}^{m}\left[b_{l} x_{i+1}^{\gamma_{l}}\right) S_{i+1}(x)\right)+(-1)^{i+2}\left(\left(b_{0}+\sum_{l=1}^{m}\left[b_{l} x_{i+2}^{\gamma_{l}}\right)\right] S_{i+2}(x)\right)\right.}{\left(*^{\prime}\right)} \\
& \left.+\ldots+(-1)^{\left(\frac{N}{2}\right)}\left(\left(b_{0}+\sum_{l=1}^{m} b_{l} x_{\frac{N}{2}}^{\gamma_{l}}\right) S_{\frac{N}{2}}(x)\right)\right] .
\end{aligned}
$$

From first part of case a) of Lemma 3.1, for (*) in interval $\left(x_{i}, x_{i+1}\right)$ we get

$\operatorname{sgn}\left((-1)^{i}\left[b_{0}\left(S_{i}(x)-S_{(i-1)}(x)\right)+\sum_{l=1}^{m}\left[b_{l}\left(x_{i}^{\gamma^{\prime}} S_{i}(x)\right.\right.\right.\right.$

$\left.\left.-x_{i-1}^{\gamma_{1}} S_{i-1}(x)\right]\right)$

$=(-1)^{i}(-1)^{\frac{N}{2}-i}=(-1)^{\frac{N}{2}}$.

The sign of other terms corresponding to pairs $\{i-3, i-2\}$, $\{i-5, i-4\}, \ldots$ is also the same as sign of $(*)$. Moreover, from second part of case a) of Lemma 3.1, it is seen that the sign of $\left(*^{\prime}\right)$ is $(-1)^{\frac{N}{2}}$, and the same argument arises for other terms corresponding to the pairs $\{i+3, i+4\}$, $\{i+5, i+6\}, \ldots$.

It should be noted that the sign of a single term that may appear like the first or the last term in the sum is also $(-1)^{\frac{N}{2}}$. Finally, the sign of $Z(x)$ is $(-1)^{\frac{N}{2}}$. When $n$ is odd, with $0 \leq \gamma_{l}^{\prime} \leq 1$, the proof is similar as $n$ even.

One should note that the above introduced weights, discussed by Theorem 3.2, include some particular weights already used by other authors. For example, with $b_{0}=1$ and $b_{l}=0 \quad \forall l \geq 1$, or with $\gamma_{l}^{\prime}=0, b_{l}^{\prime}=1$ and $b_{1}^{\prime}=0 \quad \forall l \geq 2$, the weights $u_{j}=(-1)^{j}$ are emerged that had been used by berrut [3].

By choosing $\gamma_{1}=2$ and $b_{l}=0 \forall l \geq 2$ in theorem 3.2 we get to the following corollary.

Corollary 3.2: With the conditions of theorem 3.2 and when $n$ is even, $r(x)$ with

$$
\left|u_{j}\right|= \begin{cases}b+c x_{j}^{2}, & j=0,1, \ldots, \frac{N}{2}-1, \\ \frac{1}{2}\left(b+c x_{j}^{2}\right), & j=\frac{N}{2},\end{cases}
$$

where $b, c>0$, has no real poles.

Theorem 3.3: Let $n>0$ be even and interpolation nodes satisfy (9) and $u_{j}^{\prime} s$ satisfy (10) and (11) with absolute values given by (23). Then with these weights, the barycentric rational interpolant $r(x)$ is exactly equal to $f(x)=\frac{a}{b+c x^{2}}$.
Proof: Replacing (23) in (6), considering (14) and $f$ which is an even function, then $r(x)$ is obtained as follows

$$
r(x)=\frac{a\left((-1)^{\frac{N}{2}} S_{\frac{N}{2}}(x)+S_{0}(x)+2 \sum_{j=1}^{\frac{N}{2}-1}(-1)^{j} S_{j}(x)\right)}{(-1)^{\frac{N}{2}}\left(b+c x_{\frac{N}{2}}^{2}\right) S_{\frac{N}{2}}(x)+b S_{0}(x)+2 \sum_{j=1}^{\frac{N}{2}-1}(-1)^{j}\left(b+c x^{2}{ }_{j}\right) S_{j}(x)}
$$

We investigate even and odd $\frac{N}{2}$ separately.

1. For $\frac{N}{2}$ odd, we use parts $b$ ) and $c$ ) of Lemma 3.1 to obtain

$$
\left\{\begin{array}{l}
\sum_{j=1}^{\frac{N}{2}-1}(-1)^{j}\left(b+c x_{j}^{2}\right) S_{j}(x)=\left(b+c x^{2}\right) \sum_{j=1}^{\frac{N}{2}-1}(-1)^{j} S_{j}(x), \\
-\left(b+c x_{\frac{N}{2}}^{2}\right) S_{\frac{N}{2}}(x)+b S_{0}(x)=\left(b+c x^{2}\right)\left[-S_{\frac{N}{2}}(x)+S_{0}(x)\right] .
\end{array}\right.
$$

Then from replacing these two results into the denominator of (24) we get

$$
r(x)=\frac{a\left(-S_{\frac{N}{2}}(x)+S_{0}(x)+2 \sum_{j=1}^{\frac{N}{2}-1}(-1)^{j} S_{j}(x)\right)}{\left(b+c x^{2}\right)\left(-S_{\frac{N}{2}}(x)+S_{0}(x)+2 \sum_{j=1}^{\frac{N}{2}-1}(-1)^{j} S_{j}(x)\right)}=\frac{a}{b+c x^{2}} .
$$

2. For $\frac{N}{2}$ even, we consider the denominator of (24) as follows

$$
(-1)^{\frac{N}{2}}\left(b+c x_{\frac{N}{2}}^{2} S_{\frac{N}{2}}(x)+b S_{0}(x)+2 \sum_{j=1}^{\frac{N}{2}-1}(-1)^{j}\left(b+c x_{j}^{2}\right) S_{j}(x) .\right.
$$

Like in 1, considering parts $b$ ) and $c$ ) of Lemma 3.1, and through replacing (24) the desired results is obtained.

\subsection{Practical Considerations via an Optimization Problem}

As seen in Lemma 2.1 one can transform every rational interpolant $\frac{P(x)}{Q(x)}$, to barycentric form (6). In fact, considering $\omega_{j}$ in $(3), u_{j}=\alpha \omega_{j} Q\left(x_{j}\right)$ are the desired weights, where $\alpha \neq 0$ It is now reasonable to ask whether there is just this form of weights, by which $r(x)$ equals to $\frac{P(x)}{Q(x)}$. It can be easily seen that there could be some other weights as well. For example, let us consider the following example with $\mathrm{n}=4$ for Runge function $\frac{1}{1+x^{2}}: x_{-2}=-2$, $x_{-1}=-1, x_{0}=0, x_{1}=1, x_{2}=2$ and $u_{-2}=\frac{5}{6}, u_{-1}=-\frac{4}{3}, u_{0}=1$, $u_{1}=-\frac{4}{3}, u_{2}=\frac{5}{6}$ and $u_{-2}^{\prime}=\frac{5}{2}, u_{-1}^{\prime}=-2, u_{0}^{\prime}=1, u_{1}^{\prime}=-2, u_{2}^{\prime}=\frac{5}{2}$; 
where $u_{j}=4 \omega_{j} Q\left(x_{j}\right)=4 \omega_{j}\left(1+x_{j}^{2}\right)$ and $u_{j}^{\prime}$ are obtained from (23). Replacing both sets of weights, $u_{j}$ and $u_{j}^{\prime}$ in $r(x)$ the resulting interpolant is $\frac{1}{1+x^{2}}$. Therefore, to find simple sets of weights explicitly, for which $r(x)$ in (6) is too close to a rational or non-rational function $f$, and also guaranteeing polefree rational interpolants, we consider the following optimization problem:

$$
\left\{\begin{array}{c}
\min _{u_{j}} \max _{a \leq x \leq b}|f(x)-r(x)| \\
u_{j} \text { in (22), }
\end{array}\right.
$$

where,

$$
f(x)-r(x)=\frac{\sum_{j=0}^{n}\left(u_{j}\left(f(x)-f\left(x_{j}\right)\right) \prod_{i=0, i \neq j}^{n}\left(x-x_{i}\right)\right)}{\sum_{j=0}^{n}\left(u_{j} \prod_{i=0, i \neq j}^{n}\left(x-x_{i}\right)\right)}
$$

It should be noted that solving the above optimization problem is too expensive, so we will not use that in our numerical experiments. But, our goal of solving this formula is observing the obtained $u_{j}$ for families of functions, especially rational functions, and making a connection between weights and functions. Then, we are able to explicitly give some simple weights. What we did in Theorem 3.3 for functions $\frac{a}{b+c x^{2}}$ is a case in point.

\section{Numerical Results}

We have used the MATLAB code fminimax to solve the optimization problem (25). However, the problem (25) is too expensive for computation, and as said above, solving this problem could be just helpful to see a connection between weights and functions. Therefore, in numerical results, we use $u_{j}$ in (23) when $n$ is even, and $u_{j}=(-1)^{j}\left|x_{j}\right|$. when $n$ is odd, that the weights are obtained from (22). We also use the MATLAB code of Berrut and Trefethen [9] for barycentric part. We have tested our new interpolant, denoted by "new rational", for large classes of functions, some of which are reported here. Floater and Hormann in [12] showed the strength of their interpolant with weights in (8) compared to other interpolants. We denote their method by "flo-hor" in our tables. We compare our method with "flo-hor" interpolant (with weights in (8) and $d=3$ ) as a rational interpolant, and also with cubic spline (perfect form) which is a strong piecewise interpolant, as a non-rational interpolant [10]. We take equally spaced nodes $x_{j}=-5+10 \frac{i}{n}$, for various choices of $n$ and evaluate the interpolant at 1000 equally spaced points on $[-5,5]$. In the tables "Err" denotes the mean error [17] computed by

$$
E r r=\max _{a \leq x \leq b}|f(x)-r(x)|,
$$

where, $r(x)$ is an approximation of function $f(x)$ on $[a, b]$.

\subsection{Test Problems}

Example 1. The same way we obtained the weights explicitly from which $r(x)$ becomes exactly the same as $f(x)=\frac{a}{b+c x^{2}}$ for $n$ even, one may obtain the exact weights for functions $(x)=\frac{a}{b+c x^{2 k}}$, when $k>1$. We applied the "new rational" to some of those functions to observe the results. Table 1 shows the errors in the $f(x)=\frac{1}{1+x^{4}}$ func-
tion, of the three methods.

EXAMPLE 2. We also tested the methods for some other functions. The results are seen in Tables 2 and 3 .

Table 1. Error in different interpolants for function $f(x)=\frac{1}{1+x^{4}}$

\begin{tabular}{lccc}
\hline $\mathbf{n}$ & new rational & flo-hor(d=3) & cubic spline \\
\hline 10 & $1.1 \times 10^{-1}$ & $1.2 \times 10^{-1}$ & $1.0 \times 10^{-1}$ \\
15 & $1.6 \times 10^{-2}$ & $7.0 \times 10^{-2}$ & $6.6 \times 10^{-2}$ \\
20 & $9.8 \times 10^{-3}$ & $1.0 \times 10^{-2}$ & $1.3 \times 10^{-2}$ \\
25 & $6.7 \times 10^{-4}$ & $5.8 \times 10^{-3}$ & $4.2 \times 10^{-3}$ \\
50 & $7.2 \times 10^{-5}$ & $1.4 \times 10^{-5}$ & $3.7 \times 10^{-4}$ \\
80 & $1.2 \times 10^{-6}$ & $3.2 \times 10^{-6}$ & $1.4 \times 10^{-5}$ \\
160 & $1.5 \times 10^{-9}$ & $3.1 \times 10^{-9}$ & $2.7 \times 10^{-6}$ \\
320 & $1.4 \times 10^{-10}$ & $1.9 \times 10^{-10}$ & $1.6 \times 10^{-7}$ \\
640 & $1.0 \times 10^{-11}$ & $1.3 \times 10^{-11}$ & $1.1 \times 10^{-8}$ \\
\hline
\end{tabular}

Table 2. Error in different interpolants for function $f(x)=\frac{1}{3+\cos x}$

\begin{tabular}{lccc}
\hline $\mathbf{n}$ & new rational & flo-hor(d=3) & cubic spline \\
\hline 10 & $2.2 \times 10^{-3}$ & $1.4 \times 10^{-2}$ & $3.2 \times 10^{-3}$ \\
15 & $1.9 \times 10^{-3}$ & $1.2 \times 10^{-3}$ & $9.0 \times 10^{-4}$ \\
20 & $3.6 \times 10^{-4}$ & $3.5 \times 10^{-4}$ & $2.6 \times 10^{-4}$ \\
25 & $1.2 \times 10^{-4}$ & $1.1 \times 10^{-4}$ & $9.6 \times 10^{-5}$ \\
50 & $3.1 \times 10^{-6}$ & $4.4 \times 10^{-6}$ & $4.6 \times 10^{-6}$ \\
80 & $7.2 \times 10^{-8}$ & $1.4 \times 10^{-7}$ & $3.1 \times 10^{-6}$ \\
160 & $1.5 \times 10^{-10}$ & $3.1 \times 10^{-9}$ & $2.7 \times 10^{-6}$ \\
320 & $1.4 \times 10^{-11}$ & $1.8 \times 10^{-10}$ & $1.7 \times 10^{-7}$ \\
640 & $3.5 \times 10^{-12}$ & $1.1 \times 10^{-11}$ & $1.0 \times 10^{-8}$ \\
\hline
\end{tabular}


Table 3. Error in different interpolants for function $f(x)=\frac{x^{3}}{x^{6}+4 x^{4}+5 x^{2}+2}$

\begin{tabular}{lccc}
\hline $\mathbf{n}$ & new rational & flo-hor(d=3) & cubic spline \\
\hline 10 & $3.5 \times 10^{-2}$ & $2.6 \times 10^{-2}$ & $2.3 \times 10^{-2}$ \\
15 & $2.2 \times 10^{-2}$ & $1.0 \times 10^{-2}$ & $7.5 \times 10^{-3}$ \\
20 & $3.5 \times 10^{-3}$ & $6.0 \times 10^{-3}$ & $8.5 \times 10^{-3}$ \\
25 & $3.1 \times 10^{-3}$ & $1.2 \times 10^{-3}$ & $9.6 \times 10^{-4}$ \\
50 & $7.9 \times 10^{-6}$ & $1.8 \times 10^{-6}$ & $1.0 \times 10^{-4}$ \\
80 & $2.6 \times 10^{-8}$ & $4.7 \times 10^{-7}$ & $1.0 \times 10^{-6}$ \\
160 & $1.1 \times 10^{-10}$ & $6.5 \times 10^{-10}$ & $7.6 \times 10^{-7}$ \\
320 & $2.0 \times 10^{-11}$ & $3.9 \times 10^{-11}$ & $4.6 \times 10^{-8}$ \\
640 & $1.9 \times 10^{-12}$ & $2.4 \times 10^{-12}$ & $2.9 \times 10^{-9}$ \\
\hline
\end{tabular}

\section{Conclusions}

In this paper we expressed a sufficient condition for the barycentric rational interpolation formula to have no poles when the nodes are located symmetrically relative to zero point. Then we introduced some new weights that satisfy that condition. One may concentrate on the obtained weights to find some sets of weights explicitly for different families of rational functions. We did it for special class of $\frac{a}{b+c x^{2}}$, and an explicit set of weights for odd number of nodes was given. However, we think that for even number of nodes, it is also possible to obtain the barycentric weights of this class of functions explicitly, in much the same way as odd number of nodes. Giving clues to find the simple weights by which a family of functions can be interpolated exactly is an interesting feature of this new formulation that could be considered for more investigation.

\section{References}

1. Baltensperger R, Berrut J P et al. (1999). Exponential convergence of a linear rational interpolant between transformed Chebyshev points, Mathematics of Computation, vol 68(227), 1109-1120.

2. Bellman R, Kashef B G et al. (1972). Differential quadrature: a technique for the rapid solution of nonlinear partial differential equations, Journal of Computational Physics, vol 10(1), 40-52.

3. Berrut J P (1988). Rational functions for guaranteed and experimentally well-conditioned global interpolation, Computers \& Mathematics with Applications, vol 15(1), $1-16$.
4. Berrut J P (1994). Linear rational interpolation of continuous functions over an interval, Proceedings of Symposia in Applied Mathematics, W. Gautschi, ed., AMS, Providence, RI, 261-264.

5. Berrut J P, Baltensperger R et al. (2005). Recent developments in barycentric rational interp- olation, International Series of Numerical Mathematics, vol 151, 27-51.

6. Berrut J P, and Mittelmann H (1997). Lebesgue constant minimizing linear rational interpolation of continuous functions over the interval, Computers \& Mathematics with Applications, vol 33(6), 77-86.

7. Berrut J P, and Mittelmann H (1997). Matrices for the direct determination of the barycentric weights of rational interpolation, Journal of Computational and Applied Mathematics, vol 78(2), 355-370.

8. Berrut J, and Mittelmann H D (2000). Rational interpolation through the optimal attachment of poles to the interpolating polynomial, Numerical Algorithms, vol 23(4), 315-328.

9. Berrut J P, and Trefethen L N (2004). Barycentric lagrange interpolation, SIAM Review, vol 46(3), 501-517.

10. de Boor C (2001). A practical guide to splines (Applied Mathematical Sciences), Revised Edn., Springer, vol 27.

11. Epperson J F (1987). On the Runge example, American Mathematical Monthly, vol 94(1), 329-341.

12. Floater M S, and Hormann K (2007). Barycentric rational interpolation with no poles and high rates of approximation, Numerische Mathematik, vol 107(17), 315-331.

13. Gautschi W (1997). Moments in quadrature problems, Computers \& Mathematics with Applications, vol 33(1-2), 105-118.

14. Gautschi W (1997). Numerical Analysis: An Introduction, Birkhuser, Boston.

15. Gottlieb D, Hussaini M Y et al. (1984). Theory and applications of spectral methods, Spectra Methods for Partial Differential Equations, Voigt R J, Gottlieb D, and Hussaini M Y (Eds.), SIAM, Philadelphia, 1-54.

16. Henrici P (1982). Essentials of Numerical Analysis, Wiley, New York.

17. Rivlin J C (1969). An Introduction to the Approximation of Functions, Blaisdell, Waltham, Mass.

18. Schwartz HR (1997). Numerical Analysis: A Comprehensive Introduction, Wiley, New York.

19. Stoer J, and Burlirsch R (1993). Introduction to Numerical Analysis, $2^{\text {nd }} E d n$., Springer, New York.

20. Schneider C, and Werner W (1996). Some new aspects of rational interpolation, Mathematics of Computation, vol 47(175), 285-299.

21. Taylor W J (1945). Method of lagrangian curvilinear interpolation, Journal of Research of the National Bureau of Standards, vol 35(2), 151-155. 
22. Tee $\mathrm{T} \mathrm{W}$, and Trefethen $\mathrm{L} \mathrm{N}$ (2006). A rational spectral collocation method with adaptively transformed Chebyshev grid points, SIAM Journal of Scientific Computing, vol 28(5), 1798-1811.
23. Werner W (1984). Polynomial interpolation: Lagrange versus Newton, Mathematics of Computation, vol 43(167), 205-217. 